\title{
A goal programming approach for a fuzzy single-source capacitated facility location problem
}

\author{
A. Shojaei Barjouei ${ }^{1}$, Abbas Barabadi $^{1}$, R. Tavakkoli-Moghaddam ${ }^{2}$ \\ ${ }^{1}$ Department of Technology and Safety, UiT: The Arctic University of Norway, Tromsø, Norway \\ ${ }^{2}$ School of Industrial Engineering, College of Engineering, University of Tehran, Tehran, Iran
}

(E-mail: abbas.b.abadi@uit.no)

\begin{abstract}
In this paper, we present a new mixed-integer linear goal programming (MILGP) model for a fuzzy singlesource capacitated facility location problem (F-SSCFLP), in which demands are considered as triangular fuzzy values and capacity of facilities are fuzzy constraints. At first, the basic fuzzy model for the given problem is presented. Then, utilizing goal programming (GP) approach and fuzzy principals the proposed MILGP model is introduced. Accordingly, considering a certain acceptable deviation from capacities and demands, the cost of selecting facilities and serving the demand points is minimized. Finally, to investigate the effectiveness of the presented model some large-sized problems generated randomly and all of them are solved globally in a reasonable time.
\end{abstract}

Keywords - Facility location, Mixed-integer linear goal programming, Linearization, Triangular fuzzy number.

\section{INTRODUCTION}

A single-source capacitated facility location problem (SSCFLP) deals with serving a set of demand points by a set of capacitated facilities, in which an open facility can serve one or more demand points while each demand point is served by exactly one facility under various optimization criteria [1]. This problem has extended applications in many areas, such as telecommunication, transportation and distribution systems [1-3].

To tackle with the uncertainty of the real-world situation, stochastic probabilistic theory and fuzzy theory are two main concepts. However, one of the advantages of the fuzzy theory over the stochastic probabilistic theory is its less dependency on historical data. Therefore, the fuzzy set theory, which has widely been accepted in modeling some of the vague phenomena in nature is considered in this paper.

Sakawa et al. [2] dealt with a case study of transportation planning as a facility location problem, in which both the demand and facility capacity are uncertain parameters. They considered a fuzzy goal and some constraints for the capacity of factories and demand points in the regions. Chan and Swarnkar [4] used the fuzzy goal programming model to deal with fuzzy-based multiobjective goals in a flexible manufacturing system.

In the fuzzy SSCFLP (F-SSCFLP) focused on the present work, the number of demands are triangular fuzzy values and the capacity of facilities are fuzzy constraints.

The motivation of the present work came from the study on a local delivery service of an after-sales service company which assigns its servicemen (i.e., facilities) to deliver parts and install or repair the products bought by customers in housing estates (i.e., demand points). Customers demand are fuzzy variables which must be served by just one serviceman (i.e., single-source facility). Also, the facilities should have sufficient serving capacity to cope with its demand assigned. On the other hand, servicemen have different abilities to install or repair and different capability to transport parts. Hence, it is considered as the fuzzy constraint of the capacity.

The rest of the paper is organized as follows. In Section II, after introducing notations, a fuzzy binary formulation is presented. In Section III, some principles of the fuzzy theory are reviewed. In Section IV, the proposed MILGP model for the F-SSCFLP is introduced. Section V contains some numerical examples. Finally, the paper is concluded in Section VI.

\section{FUZZY BINARY FORMULATION}

\section{A. Notations}

The notations used in problem formulation including indices, parameters and decision variables are described as follows:

\author{
Indices \\ $i \quad$ index of facilities $(i=1,2, \ldots, m)$ \\ $j \quad$ index of demand nodes $(j=1,2, \ldots, n)$ \\ Parameters \\ $f_{i} \quad$ fixed cost of facility $i$ \\ $v_{i j} \quad$ variable cost of facility $i$ while serves \\ demand node $j$ \\ $d_{j} \quad$ demand quantity of node $j$ \\ $\tilde{d}_{j} \quad$ fuzzy demand quantity of node $j$ \\ $Q_{i} \quad$ capacity of facility $i$

\section{Decision variables} \\ $Y_{i} \quad 1$, if facility $i$ is used to serving any demand \\ node; 0 , otherwise \\ $X_{i j} \quad 1$, if facility $i$ is used to serving demand node $j$; \\ 0 , otherwise
}

\section{B. Proposed fuzzy binary model}

In order to select a subset of facilities from the set $\{i=1,2, \ldots, m\}$ and find the best assignment of demand nodes to them, the following formulation is written, which is a fuzzy form of the binary model presented by Holmberg 
et al. [3] for the SSCFLP in such a way that the total cost is minimized.

$$
\begin{aligned}
& \min Z_{1}=\sum_{i=1}^{m} f_{i} Y_{i}+\sum_{i=1}^{m} \sum_{j=1}^{n} v_{i j} X_{i j} \\
& \text { s.t. } \\
& \begin{array}{ll}
\sum_{i=1}^{m} X_{i j}=1 & \forall j \\
X_{i j} \leq Y_{i} & \forall i, j \\
\sum_{j=1}^{n} \tilde{d}_{j} X_{i j} \widetilde{\leq} Q_{i} Y_{i} & \forall i \\
Y_{i}, X_{i j} \in\{0,1\} & \forall i, j
\end{array}
\end{aligned}
$$

where $\tilde{d}_{j}$ indicates fuzzy demand of node $j$ and $\widetilde{\leq}$ indicates a fuzzy constraint. Equation (1) is the objective function that minimizes the fixed costs of selecting facilities and costs of serving demand points by their assigned facility. Equation (2) guarantees each demand point is served by exactly one facility. Constraint (3) states that no demand point $j$ can be served by the facility $i$ while this facility is not selected (i.e., $X_{i j}$ cannot take the value 1 while $y_{i}=0$ ). The fuzzy constraint (4) shows the fuzzy capability of a facility to service its assigned fuzzy demands. Finally, statement (5) indicates the binary variables.

\section{SOME PRINCIPLES OF FUZZY THEORY}

In this section, we review some fundamentals of the fuzzy theory, which are used throughout this paper [5-9].

Definition 1: Consider $\tilde{A}$ as a fuzzy set in a universe of discourse $X . \quad \tilde{A}$ is described by a membership function $\mu_{A}(x)$ called the membership grade of $x$ in $\tilde{A}$. Each element $x$ in $X$ is corresponded to a real number in the range [l 1 1]. Hence, the fuzzy set $\tilde{A}$ is clearly described by:

$$
\tilde{A}=\left\{\left(x, \mu_{\tilde{A}}(x)\right) \mid x \in X\right\}
$$

Definition 2: A triangular fuzzy number $\tilde{d}_{j}$ can be defined as $d_{j}=\left(d_{j}^{1}, d_{j}^{2}, d_{j}^{3}\right)$, where $d_{j}^{k}(k=1,3)$ are the lowest and highest possible values of $d_{j}$ as shown in Fig. 1. Also, $d_{j}^{2}$ indicates the value of $d_{j}$ with the highest membership degree.

Definition 3: $\mu_{1}\left(d_{j}\right)$ is the membership function of the fuzzy number $\tilde{d}_{j}$ that achieved by:

$$
\begin{aligned}
\mu_{1}\left(d_{j}\right)=\mu_{1}\left(d_{j}^{1}\right)+S_{j, 1}\left(d_{j}-d_{j}^{1}\right)+ \\
\frac{S_{j, 2}-S_{j, 1}}{2}\left(\left|d_{j}-d_{j}^{2}\right|+d_{j}-d_{j}^{2}\right)
\end{aligned}
$$

where $|a|$ indicates the absolute value of $a$ and $S_{j, k}(k=$ $1,2)$ are the slope of left and right line piece of the triangle, respectively. The slopes are obtained by:

$$
S_{j, k}=\frac{\mu\left(d_{j}^{k+1}\right)-\mu\left(d_{j}^{k}\right)}{d_{j}^{k+1}-d_{j}^{k}} \quad k=1,2
$$

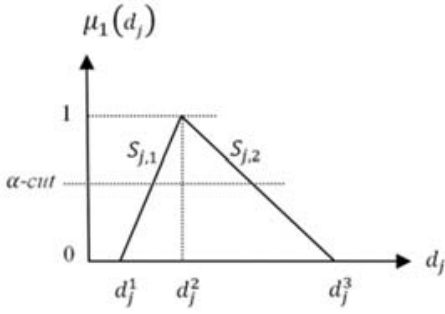

Fig. 1. Triangular membership function

Definition 4: Let $(A x)_{i} \underset{\leq}{\leq} b_{i}$ as a fuzzy constraint. The linear membership function of this fuzzy constraint is as follows:

$$
\mu_{i}(x)=\left\{\begin{array}{cl}
1 & \text { if } A x \leq b_{i} \\
1+\frac{b_{i}-(A x)_{i}}{\Delta \mathrm{b}_{i}} & \text { if } b_{i}<A x \leq b_{i}+\Delta b_{i} \\
0 & \text { if } A x>b_{i}+\Delta b_{i}
\end{array}\right.
$$

where $\Delta b_{i}$ is the tolerance related to constraint $i$ as depicted in Fig. 2. Exceeding from the constraint leads to a reduction in membership value. Then, the maximum values of $\mu_{i}(x)$ with the lowest exceed from $b_{i}$ are achieved by solving the following model [6]:

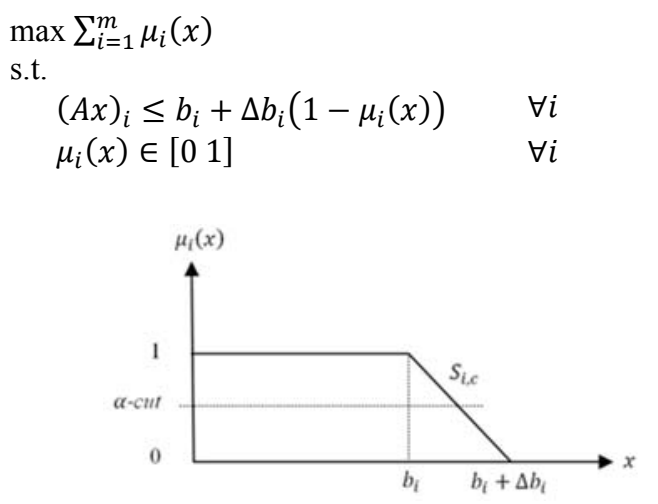

Fig. 2. Membership function of the fuzzy constraints

Definition 5: The $\alpha$-cut of the fuzzy set $\tilde{A}$ is all members of $\tilde{A}$ with the membership grade of equal or more than $\alpha \in$ $\left[\begin{array}{ll}0 & 1\end{array}\right]$ that is characterized by:

$$
\tilde{A}_{\alpha}=\left\{x \in X \mid \mu_{\tilde{A}}(x) \geq \alpha\right\}
$$

\section{CRISP MIXED-INTEGER LINEAR GOAL PROGRAMMING}

In this section, we attempt to achieve a crisp mixedinteger linear goal programming (C-MILGP) for the FSSCFLP. So, by using definitions 3 and 4, the fuzzy demands and constraints of the fuzzy binary model are transformed into their crisp values. Therefore, the crisp multi-objective mixed-integer programming model is presented below. 
$\min Z_{1}$

$\max Z_{2}=\sum_{j=1}^{n} \mu_{1}\left(d_{j}\right)$

$\max Z_{3}=\sum_{i=1}^{m} \mu_{2}\left(q_{i}\right)$

s.t.

$$
\begin{aligned}
& \sum_{j=1}^{n} d_{j} X_{i j} \leq \\
& \left(Q_{i}+\Delta Q_{i}\left(1-\mu_{2}\left(q_{i}\right)\right)\right) Y_{i} \quad \forall i \\
& \mu_{1}\left(d_{j}\right), \mu_{2}\left(q_{i}\right) \in[\alpha 1] \\
& \forall i
\end{aligned}
$$

where $Z_{1}$ was defined in (1). Also, $\mu_{1}\left(d_{j}\right), \mu_{2}\left(q_{i}\right)$ and $\Delta Q_{i}$ are membership functions of the fuzzy demands $\tilde{d}_{j}$, membership function of the $i$-th fuzzy constraint, and the tolerance related to the capacity of facility $i$, respectively. So, the membership level is maximized through (14) and (15). Variable $q_{i}$ is the used capacity of the facility $i$. Equation (16) is the crisp form of (4) using definitions 2 and 4. It is worth mentioning, $d_{j}$ is not a constant parameter, therefore the term $d_{j} X_{i j}$ in (16) is a non-linear term. Furthermore, the right-hand side of (16) is a nonlinear term. Hence, the appropriate linear form of these non-linear terms will present during this section. Also, (17) indicates the range of the membership functions, where $\alpha$ is the lowest acceptable level for membership values.

Proposition 1: Consider the following multi-objective problem:

$$
\begin{array}{ll}
\text { P1: } & \min \sum_{j=1}^{n} c_{j} x_{j} \\
& \max \sum_{j=1}^{n} \mu\left(c_{j}\right) \\
\text { s.t. } & \\
& X \in F \\
& x_{j} \in\{0,1\} \\
& c_{j} \geq 0
\end{array}
$$

where $F$ is a feasible set and membership functions are considered as objectives. An optimal solution for the model P1 is also optimal for the goal programming (GP) model $\mathrm{P} 2$ as follows:

$$
\begin{aligned}
& \text { P2: } \min \sum_{j=1}^{n} c_{j} x_{j}+\sum_{j=1}^{n}\left(w_{j}^{L} \delta_{j}^{L}+w_{j}^{R} \delta_{j}^{R}\right) \\
& \text { s.t. } \\
& \quad \mu\left(c_{j}\right)+\delta_{j}^{L}+\delta_{j}^{R}=1 \\
& \quad X \in F \\
& \quad x_{j} \in\{0,1\} \\
& \quad c_{j}, \delta_{j}^{L}, \delta_{j}^{R} \geq 0
\end{aligned}
$$

where $\mu\left(c_{j}\right)$ was defined in (7). $\delta_{j}^{L}$ and $\delta_{j}^{R}$ are deviations of $\mu\left(c_{j}\right)$ from its highest value (i.e., 1) related to left and right line segments of the membership function, respectively. Indeed, maximization of the membership functions in the model P1 is replaced by minimization of the deviations from their highest value in the model P2. According to the GP approach, $w_{j}^{L}$ and $w_{j}^{R}$ are the weights of the deviations $\delta_{j}^{L}$ and $\delta_{j}^{R}$, in a row. These weights make the appropriate change in $\mu\left(c_{j}\right)$ while $c_{j}$ changes. The weights are calculated by (19).

$$
w_{j}^{L}=\left|1 / S_{j, 1}\right|, w_{j}^{R}=\left|1 / S_{j, 2}\right| \quad \forall j
$$

Proof: To proof this proposition, we utilize the same concept used by $\mathrm{Yu}$ and $\mathrm{Li}$ [5] for a similar proposition with a maximization form of the main objective function. There is almost no single solution to optimize all the goals of the objective and membership functions simultaneously. The range of the membership functions is in the interval $\left[\begin{array}{ll}0 & 1\end{array}\right]$ while all variables and coefficients in the objective function are non-negative. Furthermore, changes in $c_{j} x_{j}$ leads to corresponding changes in left and right line segments of $\mu\left(c_{j}\right)$ accordance with $w_{j}^{L}$ and $w_{j}^{R}$, respectively. Moreover, considering the second term of the objective function in $\mathrm{P} 2$, which tries to minimize the deviations and according to the (7), $\delta_{j}^{L}$ and $\delta_{j}^{R}$ never get value simultaneously. In other words, $\delta_{j}^{L} \cdot \delta_{j}^{R}=0 ; \forall j$. So, the membership function $\mu\left(c_{j}\right)$ never gets a value more than 1. Therefore, it can be concluded that the models P1 and $\mathrm{P} 2$ are equivalent.

Proposition 2: Consider a problem with one or more absolute terms in the objective function or constraints as follows:

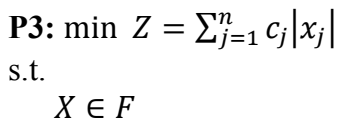

where $F$ is a feasible set, $c_{j}$ 's are given non-negative parameters and $x_{j}$ 's are unrestricted variables. Murty [10] transformed the model P3 to its equivalent linear programming (LP) model P4 by replacing $x_{j}$ and $\left|x_{j}\right|$ with $x_{j}^{+}-x_{j}^{-}$and $x_{j}^{+}+x_{j}^{-}$, respectively, where $x_{j}^{+}, x_{j}^{-} \geq 0$.

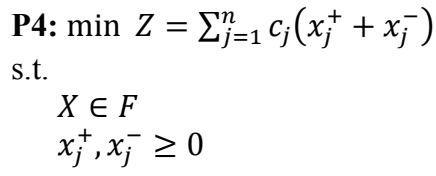

Proof: This proposition was proven by Murty [10] and we just express the results as follows:

(i) if at optimal solution $x_{j} \geq 0$, then $x_{j}^{-}=0$ and $x_{j}^{+}=x_{j}$;

(ii) if at optimal solution $x_{j} \leq 0$, then $x_{j}^{+}=0$ and $x_{j}^{-}=-x_{j}$.

They also ensured that $x_{j}^{+} \cdot x_{j}^{-}=0$.

Now, we can rewrite the linear form of (7) considering an unrestricted variable $c_{j}\left(=d_{j}-d_{j}^{2}\right)$ as follows:

$$
\begin{aligned}
\mu_{1}\left(d_{j}\right)=\mu_{1}\left(d_{j}^{1}\right) & +S_{j, 1}\left(d_{j}-d_{j}^{1}\right) \\
& +\frac{S_{j, 2}-S_{j, 1}}{2}\left(\left|c_{j}\right|+c_{j}\right)
\end{aligned}
$$


Therefore, by applying the result of Proposition 2, we have:

$$
\begin{aligned}
& \mu_{1}\left(d_{j}\right)=\mu_{1}\left(d_{j}^{1}\right)+S_{j, 1}\left(d_{j}-d_{j}^{1}\right) \\
& +\frac{S_{j, 2}-S_{j, 1}}{2}\left(c_{j}^{+}+c_{j}^{-}+c_{j}^{+}-c_{j}^{-}\right) \\
& c_{j}^{+}, c_{j}^{-} \geq 0
\end{aligned}
$$

Then, the linear form of (7) is as follows:

$$
\begin{aligned}
& \mu_{1}\left(d_{j}\right)=\mu_{1}\left(d_{j}^{1}\right)+S_{j, 1}\left(d_{j}-d_{j}^{1}\right)+ \\
& \left(S_{j, 2}-S_{j, 1}\right)\left(c_{j}^{+}\right) \quad \forall j \\
& c_{j}^{+} \geq 0 \\
& \forall j
\end{aligned}
$$

Proposition 3: Consider the following non-linear constraint:

$$
\begin{aligned}
& x y \leq b \\
& x \in\{0,1\} \\
& y \geq 0
\end{aligned}
$$

where $x$ is a $0-1$ variable, $y$ is a non-negative continues variables and $b$ is non-negative constant. Replacing the non-linear term by a new non-negative continues variable $z(=x y)$, the linear form of the above constraint is as follows:

$$
\begin{aligned}
& z \leq b \\
& y-M(1-x) \leq z \leq y+M(1-x) \\
& 0 \leq z \leq M x
\end{aligned}
$$

where $M$ is a large positive number.

Proof: this proposition can be verified as follows:

(i) if at optimal solution $x=0$, then (26) will be inactive and (27) causes $z=0$ (i.e., $z=x y=$ $0)$;

(ii) if at optimal solution $x=1$, then (27) will be inactive and (26) causes $z=y$ (i.e., $z=x y=y$ )

The proposition is proven. $\square$

Proposition 4: Consider the following binary model:

P5: $\min c x$

s.t.

$X \in F$

$x \in\{0,1\}$

$c \geq 0$

where $F$ is a feasible set. $\mathrm{P} 5$ is equivalent to $\mathrm{P} 6$ as follows:

P6: $\min y=c x$

s.t.

$y \geq c+M(x-1)$

$X \in F$

$x \in\{0,1\}$

$y, c \geq 0$

where $M$ is a large positive number.
This proposition can be proven as follows:

(i) if at optimal solution $x=1$, then $y$ will be forced to be $y=c$;

(ii) if at optimal solution $x=0$, then $y$ will be forced to be $y=0$

Thus, the proposition is proven. $\square$

Hence, the above-mentioned propositions are applied and the crisp multi-objective mixed-integer programming model is reformulated as a C-MILGP with just one linear objective. The proposed C-MILGP is as follows:

$$
\begin{array}{ll}
\min Z_{0}=\sum_{i=1}^{m} \theta_{i}+\sum_{i=1}^{m} \sum_{j=1}^{n} \varphi_{i j} & \\
\quad+\sum_{j=1}^{n}\left(w_{j}^{L} \delta_{j}^{L}+w_{j}^{R} \delta_{j}^{R}\right)+\sum_{i=1}^{m}\left(w_{i}^{c} \delta_{i}^{c}\right) & \\
\text { s.t. } & \\
\quad \sum_{j=1}^{n} \tau_{i j} \leq Q_{i}+\Delta Q_{i}\left(1-\mu_{2}\left(q_{i}\right)\right)+ & \\
M\left(1-Y_{i}\right) & \forall i \\
\tau_{i j} \geq d_{j}-M\left(1-X_{i j}\right) & \forall i, j \\
\tau_{i j} \leq d_{j}+M\left(1-X_{i j}\right) & \forall i, j \\
\tau_{i j} \leq M X_{i j} & \forall i, j \\
\mu_{1}\left(d_{j}\right)+\delta_{j}^{L}+\delta_{j}^{R}=1 & \forall j \\
\mu_{2}\left(q_{i}\right)+\delta_{i}^{c}=1 & \forall i \\
\mu_{1}\left(d_{j}\right) \geq \alpha_{1} & \forall j \\
\mu_{2}\left(q_{i}\right) \geq \alpha_{2} & \forall i \\
\theta_{i} \geq f_{i}+M\left(Y_{i}-1\right) & \forall i \\
\varphi_{i j} \geq v_{i j}+M\left(X_{i j}-1\right) & \forall i, j \\
\mu_{1}\left(d_{j}\right) \leq 1 & \forall j \\
\mu_{2}\left(q_{i}\right) \leq 1 & \forall i \\
\tau_{i j}, \theta_{i}, \varphi_{i j}, \delta_{j}^{L}, \delta_{j}^{R}, \delta_{i}^{c} \geq 0 & \forall i, j \\
\operatorname{Constraints}(2),(3),(5) \text { and }(21) &
\end{array}
$$$$
\forall i \quad(37)
$$

The objective function consists of four terms: 1) the first term and (37) are the linear form of the first term of (1) by using the proposition 4 and calculate the fixed cost of selected facilities, 2) the second term and (38) are the linear form of the second term of (1) by applying the proposition 4 and compute the variable cost of the assignments, 3) the third term and (33) are joined to calculate the deviations of the membership functions related to the fuzzy demands from their highest value (i.e., 1) by employing the proposition 1, and 4) the fourth term and (34) obtain the deviations of the membership functions related to the fuzzy constraints from their highest value (i.e., 1) by utilizing the proposition 1, in which $w_{i}^{c}=\left|1 / S_{i, c}\right|$ and $S_{i, c}$ is the slope of the right line segment of $\mu_{2}\left(q_{i}\right)$ as depicted in Fig. 2. The acceptable deviations of the membership functions from their highest value (i.e., 1) for the demands and capacities are restricted by (35) and (36), respectively. Furthermore, the linear form of (16) is achieved by uniting (29) to (32) based on proposition 3. Finally, $\mu_{1}\left(d_{j}\right)$ can be obtained from (20).

\section{NUMERICAL EXAMPLES}

In this section, to investigate the effectiveness of the proposed C-MILGP for the F-SSCFLP, a set of large-sized 
test problems comprising 200 costumers and 30 potential facilities are generated randomly $[3,6]$. The problems are as follows:

- The location of the depot is assumed to be at coordinate $(0,0)$;

- The coordinates of the location of the demand points are considered to be uniformly distributed in range $(10,200)$;

- The variable costs of facility $i$ while serves demand node $j$ are supposed to be $v_{i j}=\rho e_{i j}$, where $e_{i j}$ is the Euclidian distance between the location of the facility $i$ and the demand point $j$ and $\rho$ is a positive scalar (we assume $\rho=4$ );

- The fixed costs of facility $i$ have a uniform distribution in range $(300,700)$;

- The middle possible value of the fuzzy demands $d_{j}^{2}$ are uniformly distributed in the range of $(10,50)$; also, the lowest and highest values are determined as $d_{j}^{1}=\left(1-\lambda_{j}^{1}\right) d_{j}^{2}$ and $d_{j}^{3}=(1+$ $\left.\lambda_{j}^{3}\right) d_{j}^{2}$, where $\lambda_{j}^{1}$ and $\lambda_{j}^{3}$ are selected at random from the range $(0,1)$;

- The capacity of each facility has a uniform distribution over $(100,500)$ and the corresponding capacity tolerances are assumed to be $\Delta Q_{i}=\pi_{i} Q_{i}$ where $\pi_{i}$ is selected at random from the range $(0,1)$;

- The lowest acceptable level for the membership functions of the demand points and the facilities $(\alpha-c u t)$ are assumed $\alpha_{1}=\alpha_{2} \in$ $\{0.75,0.85,0.95\}$.

TABLE I

CPU TIMES FOR DIFFERENT LEVELS OF $\alpha-$ cut

\begin{tabular}{llll}
\multicolumn{4}{c}{ CPU TIMES FOR DIFFERENT LEVELS OF $\alpha-$ cut } \\
\hline Problem & 0.75 & 0.85 & 0.95 \\
\hline P1 & 17.75 & 9.22 & 40.3 \\
P2 & 4.81 & 7.05 & 6.5 \\
P3 & 26.78 & 27.98 & 37.36 \\
P4 & 9.2 & 6.44 & 15.02 \\
P5 & 4.5 & 7.48 & 8.39 \\
P6 & 4.38 & 5.45 & 8.28 \\
P7 & 4.95 & 5.27 & 7.53 \\
P8 & 4.52 & 8.38 & 8.61 \\
P9 & 4.11 & 4.63 & 11.58 \\
P10 & 6.33 & 6.36 & 8.53 \\
P11 & 5.75 & 5.92 & 7.83 \\
P12 & 6.33 & 7.84 & 17.13 \\
\hline
\end{tabular}

All the problems are solved globally by the CPLEX 12.9 software on a $2.40 \mathrm{GHz}$ Intel ${ }^{\circledR}$ Core $^{\mathrm{TM}}$ i5 CPU and $4 \mathrm{~GB}$ of RAM. Table I indicates the CPU times in seconds for different levels of the $\alpha$-cut. Accordingly, the $\alpha$-cut level has a direct impact on the computational time, so reducing the $\alpha$-cut level will relatively reduce the computational time. Also, the optimal values of the objective and membership functions are achieved in reasonable times which shows the strength of the proposed C-MILGP model to tackle with large-sized instances.

\section{CONCLUSION}

In this paper, fuzzy principals combined with a GP approach to deal with uncertainties of the SSCFLP in realworld. Accordingly, a new MILGP model has been presented for the F-SSCFLP, in which demands and capacity constraints were considered as triangular fuzzy numbers and fuzzy constraints, respectively. Finally, the effectiveness of the presented model has been examined trough some large-sized problems generated at random. All the test problems obtained the global optimum solutions in a reasonable time by solving the model through the CPLEX 12.9 software.

\section{REFERENCES}

[1] C.K.Y. Lin, "Stochastic single-source capacitated facility location model with service level requirements," International Journal of Production Economics, vol. 117, 2009, pp. 439-451.

[2] M. Sakawa, I. Nishizaki, Y. Uemura, "Fuzzy programming and profit and cost allocation for a production and transportation problem," European Journal of Operational Research, vol. 131, 2001, pp. 1-15.

[3] K. Holmberg, M. Ronnqvist, D. Yuan, "An exact algorithm for the capacitated facility location problems with single sourcing," European Journal of Operational Research, vol. 113, 1999, pp. 544-559.

[4] F.T.S. Chan, R. Swarnkar, "Ant colony optimization approach to a fuzzy goal programming model for a machine tool selection and operation allocation problem in an FMS," Robotics and Computer Integrated Manufacturing, vol. 22, no. 4, 2006, pp. 353-362.

[5] C.S. Yu, H.L. Li, “An algorithm for fuzzy generalized binary linear programming problems," European Journal of Operational Research, vol. 133, 2001, pp. 496-511.

[6] A.H. Gharehgozli, R. Tavakkoli-Moghaddam, N. Zaerpour, "A fuzzy-mixed-integer goal programming model for a parallel-machine scheduling problem with sequencedependent setup times and release dates," Robotics and Computer-Integrated Manufacturing, vol. 25, 2009, pp. 853-859.

[7] Y.J. Lai, C.L. Hwang, "Fuzzy mathematical programming: methods and applications," New York: Springer, 1992.

[8] H.J. Zimmermann, "Description and optimization of fuzzy systems," International Journal of General Systems, vol. 2, 1976, pp. 209-15.

[9] R.E. Bellman, L.A. Zadeh, "Decision making in a fuzzy environment," Management Science, vol. 17, 1970, pp. 141164.

[10] K.G. Murty, "Linear programming," John Wiley \& Sons, New York, 1983. 